February 2019

\title{
Chip-off Success Rate Analysis Comparing Temperature and Chip Type
}

Choli Ence

St George Police Department, ccamil2004@gmail.com

Joan Runs Through

Dixie State University, runsthrough@dixie.edu

Gary D. Cantrell

Southern Utah University, cantrellg@gmail.com

Follow this and additional works at: https://commons.erau.edu/jdfsl

Part of the Computational Engineering Commons, Data Storage Systems Commons, and the Information Security Commons

\section{Recommended Citation}

Ence, Choli; Runs Through, Joan; and Cantrell, Gary D. (2019) "Chip-off Success Rate Analysis Comparing Temperature and Chip Type," Journal of Digital Forensics, Security and Law. Vol. 13 : No. 4 , Article 7. DOI: https://doi.org/10.15394/jdfsl.2018.1545

Available at: https://commons.erau.edu/jdfsl/vol13/iss4/7

This Article is brought to you for free and open access by

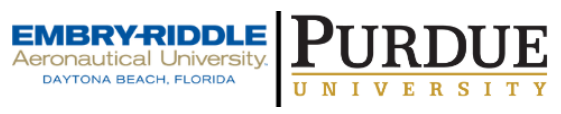
the Journals at Scholarly Commons. It has been accepted for inclusion in Journal of Digital Forensics, Security and Law by an authorized administrator of Scholarly Commons. For more information, please contact commons@erau.edu.

(c)ADFSL 


\title{
CHIP-OFF SUCCESS RATE ANALYSIS COMPARING TEMPERATURE AND CHIP TYPE
}

\author{
Choli Ence \\ St. George PD/DSU Digital Forensic Crime Lab \\ St. George, UT \\ choli.ence@dixie.edu \\ Joan Runs Through \\ DSU Digital Forensic Crime Lab \\ St. George, UT \\ runsthrough@dixie.edu \\ Gary Cantrell \\ Department of CSIS Southern Utah University \\ Cedar City, UT \\ garycantrell@suu.edu
}

\begin{abstract}
Throughout the digital forensic community, chip-off analysis provides examiners with a technique to obtain a physical acquisition from locked or damaged digital device. Thermal based chipanalysis relies upon the application of heat to remove the flash memory chip from the circuit board. Occasionally, a flash memory chip fails to successfully read despite following similar protocols as other flash memory chips. Previous research found the application of high temperatures increased the number of bit errors present in the flash memory chip. The purpose of this study is to analyze data collected from chip-off analyses to determine if a statistical difference exists between the removal temperatures of flash memory chips successfully and unsuccessfully read by using a t-test, F-test and an analysis of variance (ANOVA). The results from the statistical evaluation showed no statistical difference between the groups of memory chips successfully and unsuccessfully read, as well as, between older and newer types of Ball Grid Array (BGA) memory chips.
\end{abstract}

Keywords: digital forensics, forensics, chip-off, bit errors, physical acquisition, flash memory chip, NAND flash memory

\section{INTRODUCTION}

Digital forensics is a relatively new field of study that has drawn heavily upon the law enforcement community's effort to investigate computer related crimes (Casey, 2011, p. 10).
The digital forensic field has seen rapid changes since 2008 when the American Academy of Forensic Sciences (AAFES) officially recognized it as a science discipline and profession (Casey, 2011, p. 11). Analysis of 
mobile devices is one of the areas of digital forensic that has seen sweeping changing in the tools and even techniques used by investigators.

Mobile devices are relatively new technologies that have only been in the marketplace since the early 1980's (Goodwin, 2017). Since the introduction of the first mobile device, advancements in technology have rapidly evolved and advanced at an unprecedented rate. In fact, the introduction of new technologies quickly turns eurrent technology on the market obsolete (Yadav, 2010). Smartphone ownership has exploded since the introduction of the first smartphone in 1992 (Rainie \& Perrin, 2017). In fact, a recent Pew Research Center study found smartphone ownership now tops over 75 percent, while cellphone ownership of any kind has risen to 95 percent of the U.S. population (Pew, 2017).

These statistics are quite staggering when considering between 2011 and 2017, smartphone ownership almost doubled (Pew, 2017). Additionally, ownership of tablet computers and e-reader devices has also steadily increased over the last few years (Pew, 2017). Given this popularity of mobile devices, the law enforcement community has begun to rely heavily upon obtaining information stored on these devices to help in their investigations.

The typical forensic analysis techniques utilized by forensic investigators on small devices generally centers around the use of a plethora of hardware and software tools (Swauger, 2012). Most of these tools enable forensic investigators the ability to obtain both logical and physical acquisitions. Forensic investigators typically prefer to obtain a physical acquisition of a mobile device since it enables the recovery of unused and deleted content (Swauger, 2012). Unfortunately, forensic tools are often unable to support or fully support all the different types of cellphones on the marketplace at any given time since each make and model of cellphone requires extensive research by the vendors (Swauger, 2012). Due to the limited support, investigators are often unable to retrieve application, browser, or deleted content (Swauger, 2012).

Logical acquisitions are only capable of retrieving allocated data, which does not include the retrieval of data stored anywhere else on the memory chip (Jovanovic, 2012). In addition to limited support, typical software tools require the investigator to have the phone powered on and adjust the phone settings (Elder, 2012). Therefore, the seizure of mobile devices with password/pattern locks or physical damage, limit the tool available for use (Elder, 2012). When investigator encounter damaged, locked phones or phones not supported by current software tools, they often turn to a technique called chip-off analysis.

Chip-off analysis is a hardware-based technique used to obtain a physical acquisition of a mobile device (Jovanovic, 2012). Chip-off is the process of removing the memory chip from the circuit board using heat and then reading the memory chip utilizing a variety of adapters and chip programmers (Elder, 2012). An infrared or hot-air rework station is used to remove the memory chip from the board (Swauger, 2012). This infrared or hot-air rework station often lack accurate temperature controls and instead rely upon the training and experience of the forensic examiner to know when the temperature of the solder has reached a high enough temperature (Swauger, 2012).

Unfortunately, this brief application of heat still introduces bit errors that may prevent a successful read from occurring (Fukami, Ghose, Luo, Cai \& Mutlu, 2017). However, insufficient heat to thoroughly melt the solder connections on the memory chip may result in the removal of solder balls, and/or the solder pads from the 
memory chip, which also prevents a successfully read from occurring (Swauger, 2012).

Once the removal of the memory chip occurs, examiners must carefully clean and replace any solder balls damaged or removed during the removal process. During this process, the memory chip is heated using a hot plate to a temperature hot enough to again melt the solder (Swauger, 2012). This temperature often exceeds 220 degrees Celsius and can again introduce bit errors into the flash memory. This process is often repeated multiple times since memory chips can have between 40 to 225 individual solder points (Swauger, 2012). Additionally, the presence of epoxy on the memory chips often requires higher exposure to heat during the removal process (Swauger, 2012). The presence of the epoxy on the memory chips after removal routinely requires the application of strong chemical epoxy removers to eliminate the epoxy so the flash memory chip fits correctly into an adapter (Swauger, 2012). If the chemical epoxy remover does not fully eliminate the epoxy, the examiners must physically scrape the remaining epoxy from the chip (Swauger, 2012). Damage to the flash memory chip during this process could affect the ability to successfully read the chip.

\section{PROBLEM STATEMENT}

Although chip-off analysis allows investigations to obtain a physical acquisition when other techniques are unsuccessful, its use is not without risk. Unlike other techniques, chip-off analysis destroys the phone itself by removing the memory chip from the circuit board. Additionally, the process of removing the memory chip from the circuit board using high heat, accelerates the rate of bit errors. Inversely, the application of too little heat during the removal of the chip, damages the solder points and pads. Both scenarios present difficulties for forensic investigators since they prevent a successful read from occurring. Despite the risks associated with the use of chip-off analysis, forensic examiners routinely rely upon chip-off analysis to obtain information stored in the user data partition. Previous research conducted on the topic has primarily focused on alternative methods, as well as, as the effect architecture, seizure length, temperature and amount of program/erase cycles play in the introduction of bit errors in flash memory. Up until this point, none of the chip-off analysis research has specifically focused on determining if an ideal temperature range exists for the removal of flash memory from a circuit board to minimize the introduction of bit errors and increase the chances of obtaining a successful read. This lack of any clear temperature guidelines for the removal of flash memory chips forces forensic examiners to rely upon their training, experience and gut to determine the chip removal temperature versus an established industry wide standard.

\section{PROJECT PURPOSE}

This purpose of this study is to analyze data collected from chip-off analyses conducted at the Dixie State University Computer Crime Lab between May 2016 and February 2018 to determine if there is a statistical difference between the removal temperatures of memory chips successfully and unsuccessfully read, as well as, if there is a statistical difference between the removal temperatures of successfully read embedded Multimedia Card (eMMC) and non-eMMC flash memory chips. This paper will also discuss current research surrounding flash memory, causes for the introduction of bit errors, techniques to correct and/or mitigate the introduction of bit errors, alternative methods to thermal based chip-off analysis, and the future outlook of chip-off. Combined with the evaluation of the data, this 
study seeks to understand if there is an ideal temperature range for the removal of memory chips to maximize the ability of forensic examiners to obtain successful reads.

The study intends to prove or disprove two hypotheses using data collected from chip-off analysis conducted at the Dixie State University Computer Crime Lab over the course of 18 months between 2016 and 2018 .

-H1. There is no statistical difference between the removal temperature of memory chips successfully and unsuccessfully read.

$-\mathrm{H} 2$. There is no statistical difference between the removal temperatures of successfully read eMMC and non- eMMC memory chips.

\section{SIGNIFICANCE OF THIS STUDY}

This study intends to advance the understanding of thermal based chip-off and the role temperature plays in the ability to successfully read a chip. Examiners throughout the digital forensic field may use the results of this study to develop alternative techniques to thermal based chip-off and/or implement techniques to reduce the number of bits introduced from other sources. Results from this study may also guide future training, standard operating procedures, best practices, and policies throughout the industry both in the private and government sectors. This research contributes to the current body of knowledge and practices on this topic by specifically addressing an area of digital forensics that has received little attention.

Throughout the law enforcement communities, examiners rely upon the thermal chip-off process to obtain information otherwise not accessible from the user data partition. The thermal chip-off process is an acceptable technique but does occasionally result in unsuccessfully read memory chips.
Data stored on unsuccessfully read memory chips is inaccessible to investigators. This research provides examiners and investigators with a clear understanding of the likely causes for these bit errors along with possible techniques for mitigating them when employed the thermal chip-off process.

In addition to the data collected from actual chip-off analysis, this study includes an in-depth literature review of the existing material available on the chip-off process and the effects of temperature on the memory chips themselves. The paper also includes analysis of the data from the completed chip-off analysis to identify if an ideal temperature range exists for the removal of a memory chip based upon it being eMMC or non-eMMC. Lastly, the paper summarizes the information collected from the literature review and the research to develop recommendations on the use and implementation of chip-off analysis today, as well as, into the future.

\section{LITERATURE REVIEW}

This literature review presents current studies and research surrounding NAND flash memory and the types of bit errors present on the chip as the number of programming and erasing $(\mathrm{P} / \mathrm{E})$ cycles and temperature increases. Over the last few years, the introduction of smaller and smaller flash memory chips with ever greater memory capabilities thrusts reliability and life expectancy issues regarding the use of these chip to the forefront of the research community. Additionally, the demand for new techniques to assist in analyzing devices containing flash memory chip continues at an unparalleled pace. Several of the studies presented in this literature review discuss alternatives methods to thermal based chip-off analysis but help readers understand the importance and value of the chip-off process. Lastly, this literature review looks to the 
future success of chip-off analysis by including the current trend of encryption use in mobile devices.

\subsection{Flash Memory Storage}

Dr. Fujio Masuoka first developed flash memory for Toshiba in the early 1980's as an economical alternative to the more expensive electrically erasable programmable read-only memory (EEPROM) (Sestanj, 2016, p. 7). Unlike its predecessor, flash memory does not require a constant electrical source to store information, but rather functions with the use of floating-gate transistors to seamlessly store information throughout the memory cells (Yeh, Cheng, Chou, \& Wu, 2007, p. 1101). Flash memory functions with the use of either NAND or NOR logical gates (Sestanj, 2016, p. 7). These gates located within a transistor, hold data by storing and locking in an electrical charge for extended periods of time without the requirement for an external power supply (Breeuwsma, de Jongh, Klaver, van der Knijff, \& Roeloffs, 2007, p. 1). The parallel configuration of the memory cells within NOR flash memory, enable the random access of information within a device, making it ideal for lower-density, high-speed read applications (Toshiba, 2006, p. 2). On the other hand, the design of NAND flash memory prevents the random access of information but allows for higher density data storage in smaller cell sizes (Toshiba, 2006, p. 2). The ability for smaller cell sizes with NAND flash memory directly accounts for the ability to manufacture smaller chip designs with higher memory capacities than possible with NOR flash memory. (Toshiba, 2006, p. 2). These traits of NAND flash memory make it ideal for many devices seen in the marketplace today since they provide larger storage amounts in smaller and smaller sizes.

\subsection{NAND flash memory}

NAND flash memory stores data in cells by setting the threshold voltage, the minimum amount of voltage necessary to move a current from one cell to the next (van Zandwijk \& Fukami, 2017, p. 83). NAND flash memory consists of thousands of blocks with each block housing an array of floating gate transistor and between 32 to 64 wordlines (Cai, Haratsch, Mutlu \& Mai, 2012a). A page refers to a group of cells stored at the same physical location on cells and generally ranges in size from 2 kilobytes (KB) to $8 \mathrm{~KB}$ (Cat et al., 2012a). Increasing the numbers of electrons stored in each of the floating gate transistors, improves the overall threshold voltage (van Zandwijk \& Fukami, 2017, p. 83). Reading the data stored in each cell occurs when the threshold voltage of each cell is determined and compared against predefined values (van Zandwijk \& Fukami, 2017, p. 83). Removing all electrons from a cell and reducing the threshold voltage to zero, erases any information stored in that cell and occurs only at the block size level (van Zandwijk \& Fukami, 2017, p. 83). Although data retention in NAND flash memory occurs outside of a continuous power source, leakage of electrons from the floating gate transistors does occur over time as the oxide layers themselves degrade (Breeuwsma et al., 2007, p. $1)$.

Data retention for NAND flash memory typically falls somewhere between 10 and 100 years (Breeuwsma et al., 2007, p. 1). However, the reliability of NAND flash memory directly relates to the number of $\mathrm{P} / \mathrm{E}$ cycles, which causes electrons to move more easily through the floating gates (van Zandwijk \& Fukami, 2017, p. 83) This failure rate increases in multilevel-cell (MLC) and triple-level-cell (TLC) NAND flash memory as space between bits in a cell decrease. Both MLC and TLC architecture provide consumers with inexpensive and higher capacities storage 
options (van Zandwijk \& Fukami, 2017, p. 83). In fact, research showed $\mathrm{P} / \mathrm{E}$ cycles for MLC versus the original single-level-cell (SLC) drastically decreased from 100,000 to 3,000 cycles (Cai, et al., 2012a). In addition to decreased $\mathrm{P} / \mathrm{E}$ cycles, increased retention bit errors also occurred with MLC and TLC.

\subsection{Retention Bit Errors}

The leakage of electrons through the floating gates of a transistor results in a lower threshold voltage for the cell, which in produces erroneous results when reading the data stored in the cell since the original threshold voltage changed (van Zandwijk \& Fukami, 2017, p. 83). Retention bit errors occurred more commonly in NAND flash memory as the $\mathrm{P} / \mathrm{E}$ cycles increased (van Zandwijk \& Fukami, 2017, p. 83). Research conducted by Cai, Haratsch, Mutlu, and Mai (2012a) on NAND flash behavior error modeling found all NAND flash errors types highly correlated to the number of $\mathrm{P} / \mathrm{E}$ cycles and increased exponentially as the $\mathrm{P} / \mathrm{E}$ cycles increased. This correlation between $\mathrm{P} / \mathrm{E}$ cycles and increased errors types illustrates how the degradation in the insulation properties of the oxide layers surrounding the floating gates allowed for the leakage of more electrons through the locking gates (Cai et al., 2012a).

Research found the prevalence of retention errors rates resulted in the most frequent error of the different types of NAND flash errors and directly increased with the retention rest time itself (Cai et al., 2012a). Lastly, retention errors displayed an asymmetric nature that related directly to the value and location of the cell (Cai et al., 2012a).

This asymmetrical characteristic of retention error may prove helpful in future research when trying to find solutions for preventing or correcting these types of errors. This research provided vital information for forensic examiners across the country who conduct analysis on NAND flash memory devices seized and stored for long periods of time before initial analysis. In addition to retention bit errors, erase/program/read errors also can occur.

\subsection{Erase/program/read errors}

Like retention bit errors, erase/program/read errors all occur with some frequency in NAND flash memory. Erase errors happened when an erase function failed to return the cell back to an erased state without the presence of any stored electrons (Cai et al., 2012a). This type of error generally occurred when the oxide layers between the floating gates began to hold onto electrons as the $\mathrm{P} / \mathrm{E}$ cycles increased with use (Cai et al., 2012a). Program and read errors typically occurred when action on an adjacent cell, unintentionally programmed or altered another cell (Cai et al., 2012a). Research into NAND flash memory errors identified program errors the second most common type of error, followed by read and erase errors (Cai et al., 2012a). Program errors also displayed asymmetry but tended to shift from cells with less to more programmed electrons (Cai., et al., 2012a). This shift did not follow the typical voltage threshold shift patterns. Rather, the opposite occurred as cells with more electrons attracted even more electrons with the application of high positive programming voltage to the same world-line on other pages throughout the cell (Cai et al., 2012a). On a side note, program errors showed higher rates of occurrences on even pages than on odd pages. (Cai et al., 2012a).

Similar to program errors, read errors resulted when the threshold voltage of an adjacent cell shifted (Cai et al., 2012a). While researching this type of error, Cai et al. (2012a) found that overall propensity for this type of error demonstrated value dependence and the threshold voltages generally tended to shift towards states with higher electrons. 
Read errors manifested themselves less frequently than retention and programs errors. However, they occurred more frequently than erase errors.

Erase errors developed the least during the research and only then after the number of $\mathrm{P} / \mathrm{E}$ cycles vastly exceeded the projected lifetime of the flash memory (Cai et al., 2012a). Additionally, a reduction in the rate of erase errors occurred when a location (block) on a flash memory received consecutive erases without programming (Cai et al., 2012a). In fact, findings showed just 10 consecutive erase cycles decreased the erase errors over 95 percent (Cai et al., 2012a). This finding directly relates to the buildup of electrons within the floating gates. The application of multiple erase cycles provided more opportunities for the removal of the electrons remaining inside the floating gates (Cai et al., 2012a).

\subsection{Thermal effect on error rate}

Chip-off analysis refers to the acquisition of data directly from the flash memory of a mobile device (Ayers, Brothers \& Jansen, 2014). According to the Mobile Device Tool Classification System, chip-off analysis lies at level 4 of the pyramid, requiring extensive amounts of training and/or experience in the realm of electrical engineering, as well as, indepth understanding of file systems (Ayers et al., 2014). Furthermore, the challenges presented during the chip-off process include the risk of damaging the flash memory itself. This potential risk encourages forensic examiners to explore other less intrusive and/or risky techniques first (Ayers et al., 2014).

During chip-off analysis, forensic examiners physically remove the flash memory chip from the mobile device and then extract the data bit-for-bit directly off the chip using specialized hardware (Fukami et al., 2017). To remove the flash memory chip, the solder connecting the chip to the circuit board must melt enough to disrupt the connection between the chip and the circuit board (Fukami et al., 2017). The melting point for solder typically falls somewhere between 210 and 250 degrees Celsius (Wu, Dong, \& Zhang, 2011). The actual temperature required may fluctuate to above 250 degrees Celsius based upon the presence and the amount of epoxy. Epoxy is used in manufacturing to help secure the flash memory chip to the circuit board (Elder, 2012). The application of heat during the chipoff analysis process introduced new errors stored within the data on the chip (Fukami et al., 2017). Heating the flash memory chip to a temperature hot enough to remove it from the circuit board introduced the number of retention errors equivalent to a scenario in which a NAND flash memory chip remained untouched for over 800 years at room temperature (Fukami et al., 2017).

Given this extensive amount of retention errors, forensic examiners routinely run across unreadable chips. Clearly, this outcome presents problems for the law enforcement community who need the data stored on mobile devices to help in their investigations. However, forensic examiners often turn to chip-off analysis when other less intrusive forms of analysis prove inadequate or unable to obtain a physical acquisition of a mobile device. For example, chip-off analysis makes it possible to obtain deleted content from pattern locked devices, physically damaged devices or devices with inoperable data ports (Swauger, 2012). Some of the research conducted on this specific topic, examined the implication of the Arrhenius model on flash memory.

Throughout the industry, flash memory chip manufacturers use the Arrhenius model to determine the impact temperature plays on the aging of flash cells (Meza, Wu, Kumar, \& Mutlu, 2015). Research on the relationship of 
temperature to the failure rates of flash memory based Solid State Drives (SSDs), found failures rates of the drives increased across the platforms as temperatures increased when operating at a temperature range between 30 and 40 degrees Celsius (Meza et al., 2015). When the temperature range deviated above this range, SSDs fell into one of three categories regarding their reliability vs. temperature trends (Meza et al., 2015), The three categories included temperature-sensitive with increasing failure rate, less temperaturesensitive, and temperature-sensitive with decreasing failure rates (Meza et al., 2015).

During the research, two factors affected the role temperature played in the outcomes. In some of the SSDs, the controller attempted to regulate the temperature threshold by altering the actual activity of the processor (Meza et al., 2015). In some rare cases, the controller even shut down the SSD to prevent it from exceeding the manufactures defined threshold (Meza et al., 2015). Additionally, the presence of more than one SSD in a computer may cause the SSDs to reach higher temperatures quicker and stay longer at the higher temperatures due to a reduction in the overall airflow to each of the components (Meza et al., 2015). Not surprisingly, the results of the research found higher temperatures directly contributed to the overall increased failure rates in SSDs (Meza et al., 2015). However, research also showed SSDs utilizing throttling techniques experienced reduced failure rates. The amount of power consumption of each SSD directly correlated to the temperature of the drive itself (Meza et al., 2015). This last finding proves useful when trying to find the temperature of an SSD without an installed temperature sensor. However, the question still arises, does use of the Arrhenius model alone accurately predicts the lifetime estimation of NAND flash memory considering the other known failure mechanism.

In the research conducted by Lee et al. (2013a), use of the abnormal behavior often seen in the traditional Arrhenius model provided a more realistic lifetime estimated of NAND Flash memory versus mere reliance upon a single failure factor (p. 48). The failure mechanism believed to account for the total charge loss included de-trapping mechanism, trap-assisted tumneling mechanism, and interface trap recovery mechanism (Lee et al., 2013a, p. 48). Detrapping mechanism occurs when electrons trapped in the oxide layers shift with the application of thermal energy into the substrate (Lee et al., 2013a, p. 49). This electron shift results in an increase to the total charge loss compared to fresh cells that expand at high temperatures (Lee at al., 2013a, p, 49). Similarly, trap-assisted tunneling mechanisms also showed an overall increase as the temperatures increased causing the energy barrier between different carriers to decrease (Lee at al, 2013a, p. 49). This decrease in the energy barriers enabled carriers to pass easily through each barrier with a much lower thermal energy, which increased the rate of tumneling (Lee at al, 2013a, p. 49).

Like the other failure mechanisms, the interface trap mechanism increased with the number of $\mathrm{P} / \mathrm{E}$ cycles (Lee at al., 2013a, p. 49). However, at temperatures above 117 degrees Celsius, the interface trap recovery mechanism proved difficult to extract. Researchers eventually determined the Arrhenius model helpful in extracting information regarding the interface trap recovery mechanism (Lee at al., 2013a, p. 50). Ultimately the results from the research conducted by Lee et al. (2013a) found the careful evaluation of each type of failure mechanism yielded the most accurate results with the superposition method providing an 
accurate lifetime estimate for NAND flash memory (Lee et al., 2013a, p. 50).

Research into this topic showed generationally the cycling characteristics rate of the detrapping mechanism acted independently of previous generations since it depended strictly upon the temperature (Lee et al., 2013b, p. 1106). On the other hand, the trap-assisted tunneling mechanism strictly depended on the energy level and distance between each trap when determining the retention characteristics (Lee et al, 2013b, p. 1106). Additionally, some recent research has also examined the bit errors as a source of forensic information.

In his study, van Zandwijk (2017), exposed NAND flash chip under moderate dataretention and stress cycling to determine if similar results occurred as when exposed to extreme conditions. During the study, detectable changes in the bit error statistics occurred even when the operation of the chip fell within the specification outlined by the manufacturer (van Zandwijk, 2017). These results suggested detectable changes in the bit error statistics occurred with even limited use (van Zandwijk, 2017). Therefore, new NAND flash memory presumably responds differently than slightly used chips (van Zandwijk, 2017). This information proves especially useful in the forensic lab setting since it presented a potential method for determining the age of the device or the amount of use, as well as, the ability to correct original data by considering the number of $\mathrm{P} / \mathrm{E}$ cycles undergone by the chip (van Zandwijk 2017). In other studies, the researchers examined the relationship between the actual physical composition of the flash memory and the reliability assessment.

In the study, Govoreanu and Van Houdt (2008), compared chips manufactured with either zirconium aluminate or hafnium aluminate based interpoly dielectric, which block the leakage of electrons during the $\mathrm{P} / \mathrm{E}$ cycles. The findings of the study showed extrapolating the retention time at high temperature for the number of $\mathrm{P} / \mathrm{E}$ cycles resulted in an overestimation of the retention time (Govoreanu \& Van Houdt, 2008, p. 178). The discrepancy between the actual and predicted retention times resulted from the exclusion of possible impacts from tunnel oxide degradation (Govoreanu \& Van Houdt, 2008, p. 178). Consequently, Govoreanu and Van Houdt (2008) conducted another study which considered the trap-assisted tunneling model. The result from this new study found shallower trap levels yielded stronger temperature acceleration (Govoreanu \& Van Houdt, 2008, p. 178). Different levels of traps contributed to the overall leakage within each flash memory chip and provide valuable insight into the relationship between the composition of the interpoly dielectric and the trap levels (Govoreanu \& Van Houdt, 2008, p. 178).

Although this research focused on the composition of the interpoly dielectric, it did provide valuable insight into some of the potential factors playing into the retention and reliability issues of NAND flash memory. As illustrated throughout the research, heat drastically increased the bit error rate present in flash memory chips. Although the chip-off analysis process only heats the solder enough to melt, an exact temperature range for each type of chip does not exist. In fact, the Scientific Work Group on Digitạl Evidence (SWGDE) (2016), suggested examiners refer to the chip manufacturer's specification to obtain information on the maximum temperature for the chip. Unfortunately, manufacturers often fail to release this information or finding where to access this information proves difficult. Instead, forensic examiners routinely rely upon their gut instinct and experience to determine how much heat to apply to the chip. This often results in examiners learning to judge the best removal temperatures based upon what 
they observe on the flash memory chip and circuit board versus at a proven or standardized temperature.

Throughout this section, researchers examined various effects of the application of heat on flash memory chips. Regardless of the specific study, the research supported the assertion that the introduction of bit errors increased with an increase in temperature. To help explain this phenomenon, researchers turned to the Arrhenius model to help explain how temperature affects the aging cells within the flash memory chip. Researchers also found the chip composition, as well as the, age, and the number of $\mathrm{P} / \mathrm{E}$ cycles directly contributed to the number of bit errors present in the flash memory. Since the rate of bit errors directly correlated with the increased application of heat, researchers also examined variables that affect the amount of heat needed to remove the flash memory chip.

\subsection{Error reducing techniques for thermal based chip-off analysis}

As highlighted throughout this paper, the application of heat during thermal-based chipoff analysis greatly accelerates the rate of retention errors. In fact, research found the application of heat to a flash memory chip, generated the equivalent of between two and five years of retention errors at room temperatures (Fukami et al., 2017). This amount of retention errors can overload the error correcting abilities of the ECC and the integrity of the data itself (Fukami et al., 2017). To help combat the introduction of these retention errors during thermal based chip-off analysis, researchers studied the use of the read retry function.

In the study, researchers purposefully exposed flash memory chips to the same levels of heat used during thermal based chip-off analysis before utilizing the read retry function. The results from the use of the read retry function showed elimination of all retention errors at low $\mathrm{P} / \mathrm{E}$ cycles and the significant reduction of retention errors at high P/E cycles (Fukami et al., 2017). This research illustrates the importance of finding techniques outside of using the default read operation for continued use of thermal based chip-off analysis. However, the researcher failed to identify how to implement the read retry function inside an actual mobile device.

This topic certainly warrants additional research to determine the long-term viability of utilizing this technique to reduce bit errors. In addition to finding ways to combat the introduction of bit errors by utilizing error correcting techniques inside the flash memory chips themselves, other chip-off analysis methods exist that do not rely upon heat to remove the chip. These alternative methods to thermal based chip-off analysis significantly reduce the amount of heat directly transferred to the flash memory chip during the removal process since they do not utilize a heat source to melt the solder connecting the circuit board and the flash memory chip.

\subsection{Alternatives to thermal based chip-off analysis}

Considering the risks and increased retention errors created by the application of heat, the nonthermal chip removal process seems like a viable solution. The nonthermal chip removal process includes two different techniques referred to as the milling method and the lap and polish methods (Bair, 2018). In the milling technique, a micro milling machine slowly grids away at the circuit board on the opposite side of the flash memory chip until reaching the BGA (Billard \& Vidonne, 2015). Since the milling process stops upon reaching the BGA, no further preparation or repair of the chip itself occurs (Billard \& Vidonne, 2015). The milling techniques general takes a greater 
amount of time than the thermal based chipoff analysis with the time generally ranging between 30 minutes and an hour for the milling technique as compared to 15 to 30 minutes for the thermal chip-off process (Billard \& Vidonne, 2015). Although the time to complete the milling technique differs significantly from the thermal based chip-off analysis, it removes the introduction of retention errors (Billard \& Vidonne, 2015). Risks associated with the milling technique include unintentional milling into the flash memory chip, as well as, the potential risk to the chip from friction and/or vibration during the milling process (Bair, 2018). The other cold process technique uses water to sand away the circuit board from the flash memory chip.

The lap and polishing techniques utilize a special machine that injects water into a polishing wheel equipped with sandpaper that slowly sands away the circuit board from the back of the chip using varying degrees of sandpaper coarseness (Bair, 2018). Like the milling process, the lap and polishing techniques require constant monitoring and a time commitment to complete (Blair, 2018). This technique allows the forensic examiner to adjust the speed of the polishing wheel to keep the temperature generated during the process to a minimal amount (Blair, 2018). Like the milling technique, the lap and polishing technique takes longer than the thermal based chip-off analysis, but it limits the amount of retention errors generated during the process since the chip temperature theoretically stays within normal operating temperatures. Other alternatives to thermal based chip-off analysis include the use of flasher tools and Joint Test Action Group (JTAG) test.

An easy and noninvasive technique employed by many forensic investigators, flasher tools rely on a hardware interface to copy flash memory from a device (Breeuwsma et al., 2007). Unfortunately, use of this tool relies upon the presence of an existing interface to the data stored on the flash memory, which varies from manufacture and even device (Breeuwsma et al., 2007). Flasher tools first originated from manufacturers desire to diagnosis, debug, or update software (Breeuwsma et al., 2007). Consequently, flasher tools routinely possess functionalities outside of extracting the data stored on the flash memory chips. Although flasher tools may prove useful, they generally have limited applicability since many tools do not make a full forensic image of the flash memory or prevent the writing of data to the flash memory (Breeuwsma et al., 2007). Like flasher tools, JTAG methods utilize an existing function present in many devices to access the flash memory.

Similar to flasher tools, small personal devices do not all contain JTAG test access points. The points themselves do not grant access to stored memory, rather the process relies upon the processor granting access (Breeuwsma, 2006). If a printed circuit board (PCB) contains JTAG test access points, an image of the flash memory chip can occur using the Extest mode and a series of test vectors (Breeuwsma, 2006). The JTAG test access points development occurred in 1990 to create a standardized method for testing printed circuit boards for defects or to debug software while still in the production phase (Breeuwsma, 2006). Unfortunately, many manufacturers do not publish information on the existence or location of the JTAG test access points. In addition, JTAG test access points routinely incorporate the use of auxiliary pins spread throughout the board making use of this technique difficult and often impossible (Breeuwsma, 2006).

All these alternatives to thermal based chip-off analysis do come with some drawbacks to include limited and/or specific use and increased time requirements. Additionally, the 
researchers presenting these alternatives techniques to thermal based chip-off analysis often negatively portray other forensic techniques to increase the outlook of their technique of choice without any clear research to back their claims. This proves challenging as forensic examiners across the country argue among each other and in court on the best techniques to employ. Unfortunately, examiners and the court often turn to the technique with the best arguments without relying upon results from previously conducted studies to determine the reliability and validity of the technique. The last topic to cover in this literature review surrounds the future of chipoff analysis considering the current trend towards encryption.

\subsection{Encryption}

For the world of digital forensic, widespread use of encryption in mobile devices guarantees a tumultuous outlook for the future. Until recently, chip-off analysis provided an excellent alternative to obtaining information from damaged or locked devices (Sestanj, 2016). However, over the past few years, manufacturers have steadily introduced devices onto the marketplace with hardware encryption capabilities. In fact, Android's full disk encryption hit the market in 2011 with the introduction of Android 4.0 (Kunz, 2016). With the introduction of Android 5.0, full disk encryption shifted to the default setting on devices running this version (Kunz, 2016). Full disk encryption in Android 5.0 relied upon a single key to protect the userdata partition (Android, n.d.). Consequently, investigations conducted on devices running Android 5.0 with a secure password and encryption resulted in unsuccessful access to the device (Hintea, Bird, \& Moss, 2017).

This encryption feature changed slightly with the introduction of Android 7.0 and above, which now supports file-based encryption (Android, n.d.). Unlike full disk encryption, file-based encryption allows different keys to unlock different files completely independent of each other (Android, n.d.). Additionally, Android 7.0 included a new feature called Direct Boot that allows the device to boot directly to the lock screen (Android, n.d.). Introduction of Direct Boot proved beneficial over previous versions since a user must input their password to obtain access to any part of the disk (Android, n.d.). Use of chip-off analysis for devices running Android 7.0 and above presents problems for examiners since it includes full encryption of the userdata partition. Although the introduction of file-system encryption in Android devices only hit the market in the last few years, Apple devices included full disk encryption by default since the introduction of iOS 8 in 2014 (Apple, 2018).

To make the encryption even more secure, Apple tied the encryption key directly to the hardware of the device making brute force attacks to the recover the password, difficult at best (Apple, 2018). Consequently, attempts to access a locked Apple device prove futile even with chip-off analysis because of the presence of the encryption. Research in both Android and iOS encryption does show some promise as the law enforcement community continues to struggle to maintain access to information needed for prosecution (Kunz, 2016).

Throughout this literature review, a wide variety of research conducted on the topic surrounding chip-off analysis presented key concerns regarding its use. The primary concerns surrounding the application of heat itself involved the increased introduction of retention errors considering the errors already present in NAND flash memory. Many researchers examined various methods to reduce the errors present in flash memory to include read retry, ROR, RFR, and FCR. Although these techniques showed promising 
results, further research in this area proves necessary to truly develop techniques that completely prevent and correct the errors found in NAND flash memory. Although not the most ideal method, thermal based chip-off analysis could continue to provide a viable solution for obtaining needed information from locked and/or damaged devices when combined with error reducing techniques. However, the future use of thermal based chip-off analysis does look bleak considering the widespread use of hardware and/or file-based encryption in Android and iOS devices.

\section{METHODOLOGY}

\subsection{Hypothesis and Research Questions}

This study focuses on proving or disproving two hypotheses surrounding the effect of temperature on the ability to obtain a successful read from a memory chip removed during chip-off analysis. Data collected from the chip-off analyses conducted at the Dixie State University Computer Crime Lab between 2016 and 2018 provides a sufficient sample size to individually evaluate each null hypothesis.

-H1. There is no statistical difference between the removal temperatures of memory chips successfully and unsuccessfully read.

$-\mathrm{H} 2$. There is no statistical difference between the removal temperatures of different types of memory chip successfully and unsuccessfully read.

The selection of these two null hypotheses answers specific research questions regarding the chip-off analysis process and the application of heat. Throughout the chip-off process, the application of high levels of heat occurs during the removal of the flash memory chip form the circuit board and replacement of the solder balls. Occasionally, a flash memory chips fails to successfully read despite following similar protocols as other flash memory chips.
Since temperature plays such large role in the introduction of bit errors, the question arises regarding the temperature difference between successfully and unsuccessfully read memory chips. Additionally, since the presence of epoxy often requires the application of higher levels of heat to remove the flash memory from circuit boards, the question also arises regarding the temperature difference between flash memory with epoxy (eMMC) and those without (non-eMMC). The generation of these null hypotheses focuses on answering these questions.

The first hypothesis focuses exclusively on the independent variable of temperature regarding the dependent variable of successfully or unsuccessfully read memory chips. The second hypothesis again focuses on the independent variable of temperature and the ability to successfully and unsuccessfully read different types of memory chips, but it includes the additional dependent variable of the memory chip type (eMMC and noneMMC) to account for the presence of lack of epoxy on the flash memory chip. Each of these hypotheses utilizes the data already collected from the chip-off analysis conducted at the Dixie State University Computer Crime Lab to determine the role temperature plays in the ability to successfully read a memory chip.

\subsection{Research Design}

Examiners at the Dixie State University Computer Crime Lab collected data from 259 chip-off analyses conducted between May 2016 and January 2018, which included information regarding the memory chip manufacturer and model, type of adapter, removal temperature and the success or failure of the memory chip read. The Dixie State University Computer Crime Lab is in St. George, Utah and serves as a resource for the law enforcement community across the country. Originally started by a grant, the Computer Crime Lab now receives 
permanent funding from the State of Utah to provide local law enforcement agencies within the state of Utah with free forensic analysis for both mobile devices and computers. Beginning in 2018, law enforcement agencies outside of Utah may also continue to submit mobile devices and computer, but they do incur a nominal fee. In addition to forensic services, the Computer Crime Lab also hosts a variety of different training classes geared for the law enforcement community, which includes a class on thermal based chip-off analysis.

The data used in this study represent chipoff analyses conducted at the Computer Crime Lab between May 2016 and February 2018 and includes cellphones seized from law enforcement throughout the United States by local, state, tribal and federal authorities. Since 2010, the Computer Crime Lab has relied upon thermal based chip-off analysis to obtain information from the user data partition in damaged or locked cellphones. Unfortunately, the documentation of specific information related to the chip type, manufacture and removal temperature for the flash memory chip did not start until May 2016. The sample set for this study includes data from 259 chip-off analyses out of over 500 chip-off analyses conducted at the lab with recorded information on the chip type, manufacture and removal temperature for the flash memory chip.

\subsection{Chip-off Analysis Procedures}

All the chip-off analysis themselves occurred in a controlled environment utilizing an established chip-off protocol and an identical rework/ chip-off machine specifically designed and manufactured for the lab. The rework/chip-off machine includes an infrared temperature sensor calibrated at the time of manufacture. During each chip-off analysis, the examiner adjusted the temperature sensor to obtain a temperature reading from approximately the middle of the memory chip using either Celsius or Fahrenheit degrees. To remove the flash memory chip, the examiner slowly brought up the temperature of the flash memory chip to approximately 82 degrees Celsius using only the bottom heater on the rework/ chip-off machine. This process removed any moisture trapped between the circuit board and the flash memory chip. The examiner then slowly heated up the flash memory chip using the upper infrared lamp until the temperatures reached temperatures hot enough, between 121 and 182 degrees Celsius, to melt the solder.

Based upon the type of flash memory chip, the examiner often heated the flash memory chip above 182 degrees Celsius to melt any epoxy securing the flash memory to the circuit board. Upon removal of the flash memory chip, the examiner determined if any of the solder balls required replacement. If solder balls needed replacing, the examiner used a hot plate set to 115 degrees Celsius to heat the flash memory chip enough to again melt the solder balls. The next step in the chip-off process requires the examiner to determine the correct type of adapter needed to read the flash memory chip. The manufacturing of an adapter occurs based upon the datasheets provided/supplied by the chip manufacturers. Once the correct adapter is selected, a programmer communicates with the adapter to obtain a raw, binary dump of the flash memory chip. This raw, binary dump contains information stored in different partitions located within the flash memory chip to include the user data partition. Specialized forensic software or manual analysis presents the data into a human readable format.

For this study, some of the removal temperatures for the flash memory chip required converting to degrees Celsius to enable comparison between the analyses. Due to the limited sample size of different adapters 
type, categories included only eMMC and noneMMC flash memory chips.

\subsection{Statistical Analysis}

An excel spreadsheet containing the data obtained from each of the chip-off analysis allowed for the quantitative analysis using a tTest: two-sample assuming unequal variances, a F-test Two Sample for Variance and an analysis of variance (ANOVA). Each of these statistical analyses helped to prove or disprove the two null hypotheses regarding the statistical difference between temperatures differences between successful and unsuccessfully read and the types (eMMC and non-eMMC) of flash memory chips successfully read.

\subsection{Limitations of the Study}

All data used in this study occurred from chip-off analysis conducted at a single lab. In addition, the completion of this study resulted from an accelerated timeframe to meet the previously established deadlines for a Master's thesis. Due to this accelerated timeframe, data collection and analysis from other forensic lab located throughout the country did not make it into this study. Lastly, the limited data collection period covered for the chip-off analysis themselves, prevented the collection and analysis of larger data sets from more legacy phones using thin small outline package (TSOP) memory chips, as well as newer smartphones and tablet using universal flash storage (UFS) memory chips. This reduced sample size for both TSOP and UFS memory chips, limited the results obtained.

The examiners employed at Dixie State University Computer Crime Lab possess similar training, experience, and certification as other professionals this the field. The author conducted many of the chip-off analyses used in this study, which may result in some confirmation bias during the data interpretation.

\section{RESULTS}

\subsection{Survey Demographics}

The study consisted of data from 259 chip-off analyses conducted at the Dixie State University Computer Crime lab between May 2016 and February 2018. Of those chip-off analyses, only 14 unsuccessful analyses resulted. The temperature distributions for chips successfully and unsuccessfully read are illustrated in Figures 1-2. The mean temperature for the successful chip-off analysis was 221.63 degrees Celsius, while the mean temperature for the unsuccessful chip-off analysis was 221.60 degrees Celsius. The mean temperatures for both the successful and unsuccessful chip-off analysis were indicated by the red lines in Figures 1-2. The distribution of BGA flash memory chips used in the study that were eMMC were significantly higher than non-eMMC $(16 \%$ eMMC and $84 \%$ noneMMC.) $95 \%$ of reads were successful and $5 \%$ were unsuccessful. 


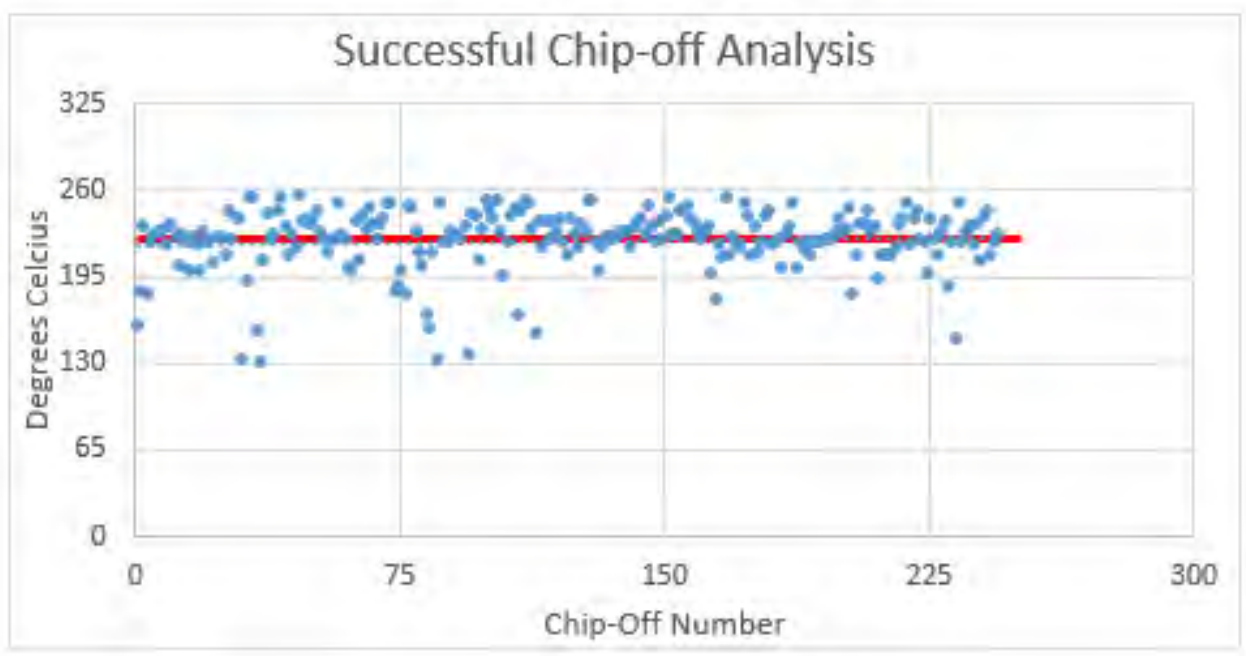

Figure 1. Temperature distribution of chips successfully read.

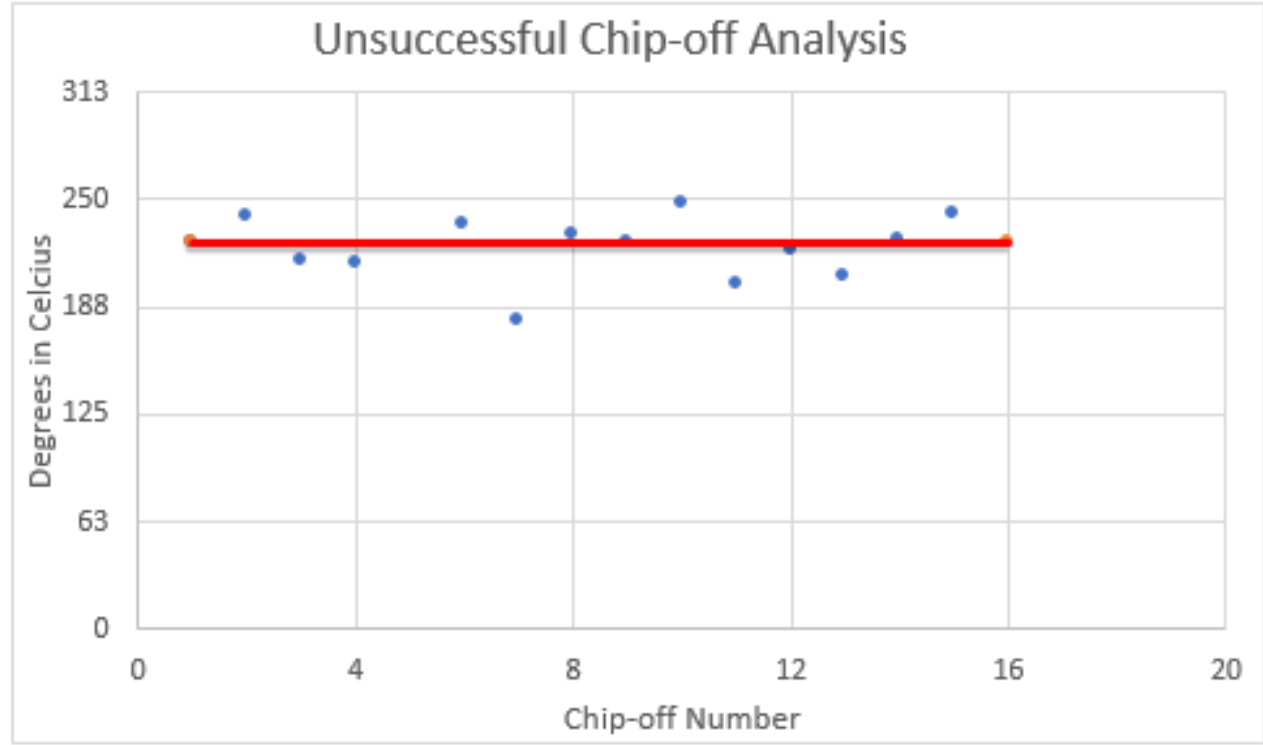

Figure 2. Temperature distribution of chips unsuccessfully read.

\subsection{Impact of temperature}

The data obtained for the successful and unsuccessful chip-off procedures were statistically evaluated to determine if the temperature variance between the two groups were equal. The F-test Two Sample for Variance was calculated based upon the null hypothesis that there is no statistical difference 
between the removal temperatures of memory chips successfully and unsuccessfully read. The alternative hypothesis of there is a statistical difference between the removal temperatures of the memory chips successfully and unsuccessfully read. As illustrated in Table 1, F $(1.51)<$ F critical one tail (2.22) and P (0.199) $>0.05$. A t-Test: two-sample assuming unequal variances was also calculated to determine whether there is a statistical difference between the means of the memory chips successfully and unsuccessfully read. For the t-test, the null and alternative hypotheses remained the same as in the F-test. Table 2 provides the results of the t-test with $\mathrm{P}(0.199)>0.05$ and the t-value $(0.0077)<$ t-critical value two tail (2.13). Lastly, an analysis of variance (ANOVA): single factor was used to again compare the means between the successful and unsuccessful read memory chips. The results of the ANOVA showed the p-value $(0.99)>.05$ and $\mathrm{F}$ $(0.000041)<\mathrm{F}$ crit (3.88) as illustrated in Table 3. The results from the F-test, t-test and ANOVA all resulted in the null hypothesis not being rejected as there was not a statistical difference between the temperatures of memory chips successfully and unsuccessfully read.

Table 1

Statistical evaluation using the F-test Two Sample for Variance to compare memory chips successfully and unsuccessfully read.

F-Test Two-Sample for Variances

\begin{tabular}{lrr}
\hline & Variable I & Variable 2 \\
\hline Mean & 221.6349 & 221.5952 \\
Variance & 515.655 & 340.5178 \\
Observations & 245 & 14 \\
df & 244 & 13 \\
F & 1.514326 & \\
P(F $<=$ f) one-tail & 0.199418 & \\
F Critical one-tail & 2.229286 & \\
\hline
\end{tabular}


Table 2

Statistical evaluation using the t-Test: two-sample assuming unequal variances to compare memory chips successfully and unsuccessfully read.

\begin{tabular}{|c|c|c|}
\hline \multicolumn{3}{|c|}{ t-Test: Two-Sample Assuming Unequal Variance } \\
\hline & $\begin{array}{c}\text { Variable } \\
1\end{array}$ & $\begin{array}{c}\text { Variable } \\
2\end{array}$ \\
\hline Mean & 221.6349 & 221.5952 \\
\hline Variance & 515.655 & 340.5178 \\
\hline Observations & 245 & 14 \\
\hline $\begin{array}{l}\text { Hypothesized Mear } \\
\text { Difference }\end{array}$ & \multicolumn{2}{|l|}{0} \\
\hline df & \multicolumn{2}{|l|}{15} \\
\hline t Stat & \multicolumn{2}{|l|}{0.007719} \\
\hline$P(T<=t)$ one-tail & \multicolumn{2}{|l|}{0.496971} \\
\hline t Critical one-tail & \multicolumn{2}{|l|}{1.75305} \\
\hline $\mathrm{P}(\mathrm{T}<=\mathrm{t})$ two-tail & \multicolumn{2}{|l|}{0.993943} \\
\hline t Critical two-tail & \multicolumn{2}{|l|}{2.13145} \\
\hline
\end{tabular}


Table 3

An analysis of variance (ANOVA) single factor comparing the means of memory chips successfully and unsuccessfully read.

\section{Anoxa: Single Factor}

SUMMARY

\begin{tabular}{lrrrr}
\hline Groups & Count & Sum & Average & Variance \\
\hline Column 1 & 245 & 54300.56 & 221.6349 & 515.655 \\
Column 2 & 14 & 3102.333 & 221.5952 & 340.5178
\end{tabular}

ANOVA

\begin{tabular}{lccccccc}
\hline $\begin{array}{c}\text { Source of } \\
\text { Variation }\end{array}$ & SS & $d f$ & MS & $F$ & P-value & F crit \\
\hline Between Groups & 0.020854 & & 1 & 0.020854 & $4.11 \mathrm{E}-05$ & 0.994887 & 3.877896 \\
Within Groups & 130246.6 & & 257 & 506.7959 & & & \\
& & & & & & & \\
& & & & & & & \\
Total & 130246.6 & 258 & & & & \\
\hline
\end{tabular}

\subsection{Impact of Chip type}

The data obtained from the successful and unsuccessful chip-off procedures were statistically evaluated to determine if a statistical difference exists between the removal temperatures of different types of memory chips successfully read. Due to incomplete data collection, some chip-off analyses were excluded. The F-test two sample for variance was calculated based upon the null hypothesis there is no statistical difference between the removal temperatures of eMMC and non-eMMC memory chips successfully read. As illustrated in Table 4, F (1.58) $<$ F critical one tail (1.60) and $\mathrm{P}(0.06)>0.05$ showed the variations between the two samples were equal and the null hypothesis could not be rejected. The t-test two sample assuming unequal variances was calculated using the same null hypothesis as used in the F-test. Table 5 lists the results from the t-test with $\mathrm{P}$ $(0.136)>0.05$ and the t-value $(1.11)<\mathrm{t}$ critical value two tail (2.00). Again, the results of the t-test cannot reject the null hypothesis. Finally, an analysis of variance (ANOVA): 
single factor was used to compare the means between the eMMC and non-eMMC types of memory chips. The results of the ANOVA showed the p-value $(0.34)>.05$ and $\mathrm{F}(0.90)$
$<\mathrm{F}$ crit (3.88) as illustrated in Table 6. Like the previous statistical evaluations, the results of the ANOVA could also not reject the null hypothesis.

Table 4

Statistical evaluation using the F-test Two Sample for Variance to compare types of memory chips successfully and unsuccessfully read.

\begin{tabular}{|c|c|c|}
\hline \multicolumn{3}{|c|}{ F-Test Two-Sample for Variances } \\
\hline & Variable I & Variable 2 \\
\hline Mean & 222.332139 & 218.3734568 \\
\hline Variance & 555.442775 & 352.5538997 \\
\hline Observations & 186 & 36 \\
\hline df & 185 & 35 \\
\hline F & 1.57548328 & \\
\hline$P(F<=f)$ one-tail & 0.05587099 & \\
\hline F Critical one-tail & 1.60067569 & \\
\hline
\end{tabular}


Table 5

Statistical evaluation using the t-Test: two-sample assuming unequal variances to compare types of memory chips successfully read.

\begin{tabular}{|c|c|c|}
\hline \multicolumn{3}{|c|}{ t-Test: Two-Sample Assuming Unequal Variances } \\
\hline & Variable 1 & Variable 2 \\
\hline Mean & 222.332139 & 218.3734568 \\
\hline Variance & 555.442775 & 352.5538997 \\
\hline Observations & 186 & 36 \\
\hline $\begin{array}{l}\text { Hypothesized Mea } \\
\text { Difference }\end{array}$ & \multicolumn{2}{|l|}{0} \\
\hline df & \multicolumn{2}{|l|}{59} \\
\hline t Stat & \multicolumn{2}{|l|}{1.10737599} \\
\hline $\mathrm{P}(\mathrm{T}<-t)$ one-tail & \multicolumn{2}{|l|}{0.13631287} \\
\hline t Critical one-tail & \multicolumn{2}{|l|}{1.67109303} \\
\hline $\mathrm{P}(\mathrm{T}<-\mathrm{t})$ two-tail & \multicolumn{2}{|l|}{0.27262574} \\
\hline t Critical two-tail & \multicolumn{2}{|l|}{2.00099538} \\
\hline
\end{tabular}


Table 6:

An analysis of variance (ANOVA) single factor comparing the means of different types of memory chips successfully read.

\begin{tabular}{|c|c|c|c|c|}
\hline \multicolumn{5}{|c|}{$\begin{array}{l}\text { Anoxa: Single } \\
\text { Factor }\end{array}$} \\
\hline \multicolumn{5}{|l|}{ SUMMARY } \\
\hline Groups & Count & Sum & $\begin{array}{c}\text { Averag } \\
e\end{array}$ & $\begin{array}{c}\text { Varian } \\
\text { ce }\end{array}$ \\
\hline eMMC & 186 & $\begin{array}{r}41353.777 \\
78\end{array}$ & $\begin{array}{r}222.33 \\
21\end{array}$ & $\begin{array}{r}555.44 \\
28\end{array}$ \\
\hline non-eMMC & 36 & $\begin{array}{r}7861.4444 \\
44\end{array}$ & $\begin{array}{r}218.37 \\
35\end{array}$ & $\begin{array}{r}352.55 \\
39\end{array}$ \\
\hline
\end{tabular}

ANOVA

\begin{tabular}{lrrrrrr}
\hline \multicolumn{1}{c}{$\begin{array}{c}\text { Source of } \\
\text { Variation }\end{array}$} & \multicolumn{1}{c}{ SS } & df & MS & $F$ & value & $F$ crit \\
\hline & 472.6761 & & 472.67 & 0.9034 & 0.3428 & 3.8840 \\
Between Groups & 17 & & 61 & 93 & 9 & 75 \\
& & & 523.16 & & & \\
Within Groups & 115096.3 & 220 & 5 & & & \\
& & & & & & \\
& 115568.9 & & & & & \\
Total & 76 & 221 & & & & \\
\hline
\end{tabular}

\section{DISCUSSION OF RESULTS}

The statistical evaluation of the data obtained from the chip-off analysis conducted at the Dixie State University Computer Crime Lab helped to confirm many of the assumptions laid out in the hypotheses. This evaluation also helped to solidify some of the previous research conducted on this topic regarding the use of the thermal chip-off process and the introduction/presence of bit errors in flash memory as presented in the literature review.

Though the amount of data from memory chips unsuccessfully read included in this study were low (5 percent), it still contained a large 
enough sample size to use for comparison against memory chips successfully read. The mean temperatures for both successfully and unsuccessfully read memory chips fell within .03 degrees Celsius of each other. During statistical evaluation, this difference proved to not be statistically different using either a Ftest, t-test or ANOVA. Additionally, when examining the scatter plot from both groups, the temperatures primarily fell between 150 and 250 degrees Celsius. Those few chip-off analyses that feel below the temperature range of 150 degrees Celsius were minimal in number and generally associated with non-eMMC memory chips affixed to the circuit board without the use of epoxy. These lower temperatures were not unexpected since the lack of epoxy on the memory chips results in reduced temperatures for the memory chip removal.

To determine if these lower temperatures seen in non-eMMC memory chips were significantly different than those temperatures seen in eMMC memory chips, the groups were evaluated using the F-test, t-test and ANOVA. Again, the results of the statistical evaluation found no statistical difference between the two groups. This finding is surprising especially when considering the research from the literature review found an increase in the introduction rate of bit errors when the temperatures increased.

\section{SUMMMARY AND RECOMMMNDATIONS}

The use of digital forensics by law enforcement has steadily increased since the introduction of the first mobile device into the marketplace. Many different software and hardware options are now available to analyze the majority of digital devices. Occasionally, the presence of passcode/pattern locks or damages to the digital devices, prevent the use of typical software and hardware options. Thermal chip- off analysis has become a viable option for gaining access to the user data stored on digital devices that would otherwise not be accessible. The data used in this research was collected from the Dixie State University Computer Forensic Lab where thermal chip-off analysis is conducted for law enforcement agencies across the county. The literature review used in this research was primarily focused on the possible causes of bit errors in flash memory and viable alternatives to the thermal chip-off process

The results of the statistical evaluation of the data collected from the flash memory chip's unsuccessful and successful read found the two groups were the same. The same results occurred when comparing eMMC and non-eMMC flash memory.

The data used for this research was collected from a single forensic lab. Future research should elicit data from different forensic lab in order to increase the sample size of memory chips unsuccessfully read. Additionally, research comparing the thermal and non-thermal based chip-off analysis could help to determine if there a statistical advantage exist from of one type of process over the other.

In the coming years, the introduction of encrypted devices from the manufactures could prove problematic for forensic examiners relying upon chip-off analysis to obtain information stored in the user data partition. Continued research needs to continue by both law enforcement and digital forensic communities to come up with viable solutions to defeat the new security features introduced by manufactures. In the meantime, locked or damaged devices currently running Android 7.0 or Apple iOS 8 and above prove problematic for examiners to gain access to the user data partition. Unfortunately, manufacturers quickly work to repair known or discovered vulnerability in the system resulting 
in the limited publication or dissemination of this information. Some vendors such as Cellebrite report finding work arounds to defeating hardware encryption and offer this service to customers at the cost of thousands of dollars per device.

Although this technique certainly reduces the chances of manufactures repairing or closing an identified vulnerability, it greatly limits the number of devices examined by law enforcement to only the most serious crimes. In this digital age, limiting data collection to only the most serious crimes places many citizens throughout the country at risk and reduces the effectiveness of the legal system as a whole. Collaborative efforts between both private and govermment agencies must continue into the future to ensure continued access to data legally authorized to obtain by the legal system. 


\section{REFERENCES}

Android. (n.d.). Encryption. Retrieved from https://source.android.com/security/encry ption/

Apple. (2018). iOS security Guide. Retrieved from

https://www.apple.com/business/docs/iOS _Security_Guide.pdf

Ayers, R., Brothers, S., \& Jansen, W. (2014). Guidelines on mobile device forensics (NIST Special Publication 800-101). Washington, DC: U.S. Department of Commerce.

Bair, J. (2018). Seeking the truth from mobile evidence: Basic fundamental, intermediate and advanced overview of current mobile forensic investigations. San Diego, CA: Elsevier.

Billard, D., \& Vidonne, P. (2015). Chip-off by matter subtraction: Firgida Via. Conference on Systematic Approaches to Digital Forensic Engineering.

Breeuwsma, M. F. (2006). Forensic imaging of embedded systems using JTAG (boundaryscan). Digital Investigation, 3, 32-42.

Breeuwsma, M, de Jongh, M., Klaver, C., van der Knijff, R., \& Roeloffs, M. (2007). Forensic data recovery from flash memory. Small Scale Digital Device Forensics Journal, 1(1).

Cai, Y., Haratsch, E. F., Mutlu, O., \& Mai, K. (2012a). Error patterns in MLC NAND flash memory: Measurement, characterization, and analysis. Design, Automation \& Test in Europe Conference \& Exhíbition.

Cai, Y., Haratsch, E. F., Mutlu, O. \& Mai, K. (2013). Threshold voltage distribution in
MLC NAND flash memory: Characterization, analysis, and modeling, Design, Automation \& Test in Europe Conference \& Exhibition.

Cai, Y., Mutlu, O, Haratsch, E, F., \& Mai, K. (2013). Program interference in MLC NAND flash memory: characterization, modeling, and mitigation. IEEE 31st International Conference on Computer Design.

Cai, Y., Luo, Y., Haratsch, E. F., Mai, K., \& Mutlu, O. (2015). Data retention in MLC NAND flash memory: characterization, optimization, and recovery. IEEE Symposium on High Performance Computer Architecture.

Cai, Y., Yalcin, G., Mutlu, O., Haratsch, E. F., Cristal, A., Unsal, O. S., \& Mai, K. (2012b). Flash correct-and-refresh: Retention-Aware error management for increased flash memory lifetime. IEEE International Conference on Computer Design, 94-101.

Casey, E. (2011). Digital evidence and computer crime: Forensic science, computers and the internet (3rd ed.).

Waltham, MA, Academic Press.

Chang, Y.H., Wu, P. L., Kuo, T. K. \& Hung, S. H. (2012). An adaptive file-systemoriented FTL mechanism for flash-memory storage systems. ACM Transactions on Embedded Computing System, 11(1).

Choi, H., Liu, W., \& Sung, W. (2010). VLSI implementation of $\mathrm{BCH}$ error correction for multilevel cell NAND flash memory. IEEE Transactions on Very Large-Scale Integration (VLSI) Systems, 18(5), 843847. 
Elder, B. (2012). Chip-off and jtag analysis for mobile device forensics. Evidence Technology, 10(3).

Fukami, A., Ghose, S., Luo, Y., Cai, Y., \& Mutlu, O. (2017). Improving the reliability of chip-off forensic analysis of NAND flash memory devices. Digital Investigation, 20, S1-S11.

Goodwin, R. (2017). The history of mobile phones from 1973 to 2008: The handsets that made it all happen. Retrieved from http://www.knowyourmobile.com/nokia/n okia-3310/19848/history-mobile-phones1973-2008-handsets-made-it-all-happen

Guenin, B. M. (1997). Packing: designing for thermal performance. Electronic Cooling, $3(2), 14-19$.

Govoreanu, B., \& Van Houdt, J. (2008). On the roll-off of the activation energy plot in high-temperature flash memory retention test and its impact on the reliability assessment. IEEE Electron Device Letters, $20(2), 177-179$.

Hintea, D., Bird, R., \& Moss, J. (2017). An investigation into identifying password recovery and data retrieval in the android operating system. Proceedings of the 16th European Conference on Cyber Warfare and Security ECCWS 2017, 165-171.

Jovanovic, Z. (2012). Android forensic techniques. International Academy of Design and Technology, Retrieved from http://www.bulleproof.com/Papers/Androi d\%20Forensies\%20Techniques.pdf

Kunz, O. (2016). Android full-disk encryption: A security assessment (Master's thesis). Retrieved from https://www.royalholloway.ac.uk/

Lee, K., Kang, M., Seo, S., Li, D. H., Kim, J., \& Shin, H. (2013a). Analysis of failure mechanisms and extraction of activation energies (Ea) in 21-nm NAND flash cells. IEEE Electron Device Letter, 34(1), 48-50.

Lee, K., Kang M., Seo, S., Kang, D., Kim, S., Li, D. H., \& Shin, H. (2013b). Activation energies (Ea) of failure mechanisms in advanced NAND flash cells for different generations and cycling. IEEE Transaction on Electron Devices, 60(3), 1099-1107.

Meza, J., Wu, Q., Kumar, S., \& Mutlu, O. (2015). A large-scale study of flash memory failure in the field. Sigmetrics.

Pew Research Center. (2017). Mobile fact sheet. Retrieved from http://www.pewinternet.org/factsheet/mobile/

Rainie, L. \& Perrin, A. (2017). 10 facts about smartphones as the iPhone turns 10. Pew Research Study. Retrieved from http://www.pewresearch.org/facttank/2017/06/28/10-facts-aboutsmartphones/

Regan, J. E. (2009). The forensic potential of flash memory (Master's thesis). Retrieved from Defense Technical Information Center. (Accession No. ADA509258).

Sestanj, I. (2016). NAND flash data recovery cookbook. Belgrade, Serbia: Author.

Swauger, J. (2012) Extracting a full bit-stream image from devices containing embedded flash memory. Digital Forensics. Recovered from http:/ www.binaryintel.com/wpcontent/uploads/2012/05/Chip-

Off_Forensics_Article.pdf

SWGDE. (2016). SWGDE best practices for chip-off (Version 1.0). Scientific Working Group on Digital Evidence. Retrieved from

https://www.swgde.org/documents/Curren t\%20Documents/SWGDE $\% 20$ Best $\% 20$ Prac tices $\% 20$ for $\% 20$ Chip-Off 
Toshiba. (2006). NAND vs. NOR flash memory: Technology overview. Retrieved from

http://aturing.umcs.maine.edu/ ${ }^{\sim}$ meadow/ courses/cos335/

Toshiba\%20NAND_vs_NOR_Flash_Memory _Technology_Overviewt.pdf

Toshiba. (2016). Flash memory: SemiConductor \& storage products. Retrieved from http://toshiba.semicon-storage.com/

van Zandwijk, J. P. (2107). Bit-errors as a source of forensic information in NANDflash memory. Digital Investigations, 20, S12-S19.

Van Zandwijk, J. P., \& Fukami, A. (2017). NAND flash memory forensic analysis and the growing challenge of bit errors. IEEE Computer and Reliability Societies.

Wu. Q., Dong, G., Zhang, T. (2011). Exploiting heat-accelerated flash memory wear-out recovery to enable self-healing SSDs. Proceedings of the Workshop on Hot Topics in Storage and File Systems.

Yadav, A. (2010). How long does it take for technology to become outdated? Technolism. Retrieved from http://www.technolism.com/new-oroutdated-how-fast-the-latest-technologybecomes-old-in-todays-world.html

Yeh, J., Cheng, K., Chou, Y., \& Wu, C. (2007). IEEE Transactions on ComputerAided Design or Integrated Circuits and Systems, 26(6), 1101-1113. 